\title{
Heart failure as an endpoint in hypertension trials?
}

Paul K. Whelton, MB, MD, MSc

Tulane University School of Public Health and Tropical Medicine,

Tulane University School of Medicine

The importance of heart failure as a cardiovascular complication, the central role of blood pressure as the most important preventable risk factor for heart failure, and the demonstrated efficacy of antihypertensive drug therapy for prevention of heart failure combine to make a compelling case for inclusion of heart failure in antihypertensive therapy event-based trials, especially those conducted in older adults.

\section{Prevalence and burden of heart failure}

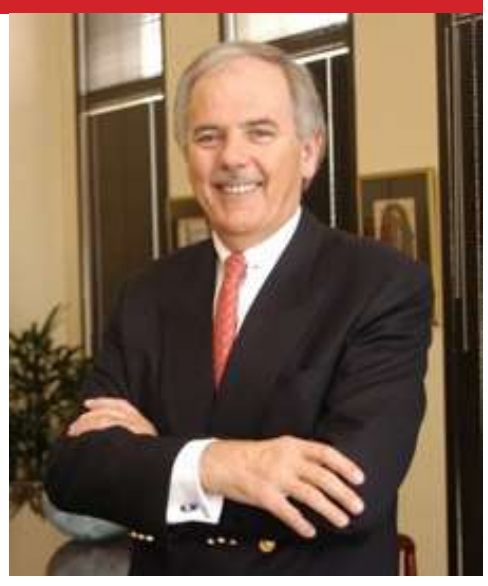

Heart failure is an increasingly common cardiovascular complication that has serious consequences for the individual and for society, especially in high-income countries like the United States (US). In contrast to the overall trend for a progressive decline in cardiovascular disease mortality among US adults, heart failure prevalence is increasing ${ }^{1}$. Based on data from the US National Health and Nutrition Examination Survey, approximately 6.2 million US adults had heart failure in 2013-2016. Heart failure is very age dependent, with lifetime risks varying from 20-45\% in adults 45 years or older, depending on age and ethnicity. In Framingham Study analyses, heart failure has been about eight times more common in the eight compared to the fifth decade of life. Approximately $80 \%$ of the hospitalizations due to heart failure and $90 \%$ of heart failure-related deaths occur in adults aged 65 years or older. The US Census Bureau predicts this segment of the population will almost double in size, to approximately 80 million persons (about 20\% of the population) by 2050. Because of this change in demography and better survival from incident heart failure as well as other diseases, the prevalence of heart failure is likely to increase substantially unless curbed by prevention interventions. Current estimates suggest an increase to more than 8 million US adults with heart failure by 20301. US population estimates identify current annual expenditure for heart failure to be between $\$ 30$ to $\$ 40$ billion. Much of the economic burden results from hospitalization expenses, followed by costs related to drug purchases, office visits and lost productivity. Worldwide heart failure annual costs have been estimated to approximate $\$ 110$ billion but the financial burden is expected to increase substantially in future years.

\section{Blood pressure and heart failure}

High blood pressure is the most important modifiable risk factor for heart failure. In the Prospective Studies Collaborative meta-analysis of 61 cohorts (almost 1 million participants) there was a log-linear relationship between usual systolic as well as diastolic blood pressure and heart failure mortality, with no evidence of a threshold in risk down to at least $115 / 75 \mathrm{~mm} \mathrm{Hg}^{2}$. Likewise, in a linked electronic health record study of approximately 1.25 million adults a 20/10 mm Hg higher level of systolic/diastolic blood pressure was associated with a heart failure events hazard ratio ( $95 \%$ confidence interval) of 1.27 (1.23 to 1.32)/1.23 (1.14 to 1.29), with no evidence of a threshold in risk $^{3}$. In addition to this direct relationship, high blood pressure is the underlying cause of left ventricular hypertrophy and other heart failure risk factors such as ischemic heart disease ${ }^{4}$.

\section{Heart failure in clinical trials}

Based on its epidemiology, lowering blood pressure should prevent heart failure, especially in older adults. Consistent with this expectation, there was no incident heart failure in the actively treated group compared to 11 such events in the placebo group in the 1970 Veterans Administration Cooperative Study Group trial of antihypertensive, despite the fact that their mean age was only 50 years ${ }^{5}$. One would expect heart failure to be a more prominent feature in trials with older adults. The heart failure experience in six trials of antihypertensive drug treatment conducted in older adults is displayed in the table. In each trial, heart failure was less common in the group assigned to more intensive blood pressure reduction. Consistent with biology, the reduction in heart failure was most prominent in HYVET (64\% with a 95\% Cl 42-78\%; $\mathrm{p}<0.001$ ), a placebo-controlled trial conducted in a cohort with a mean age of 83 years at baseline $^{6}$. However, even in the active treatment-controlled Systolic Blood Pressure Intervention Trial (SPRINT) more intensive antihypertensive drug treatment resulted in a $38 \%$ reduction in heart failure (hazard ratio 0.62 and $95 \% \mathrm{Cl}$ 0.45 to $0.84 ; p=0.002$ ). 
Heart failure is a very relevant trial outcome with serious health consequences. In the Systolic Blood Pressure Intervention Trial (SPRINT), diagnosis of new onset heart failure was associated with a 9.5 fold higher risk of death from any cause, a 26.8 fold increase in death from cardiovascular disease, a 15.7 fold increase in myocardial infarction, a 9.9 fold increase in non-myocardial infarction acute coronary syndrome, and a four-fold increase in stroke during the remainder of trial follow-up 7 . In addition, almost one-third (29\%) of those with new onset ADHF had a second episode of heart failure during follow-up. Heart failure in the Antihypertensive and Lipid-Lowering Treatment to Prevent Heart Attack Trial (ALLHAT) was also associated with a markedly increased risk of mortality, independent of treatment group allocation, both during the trial ${ }^{8}$ and during extended follow-up for an average of 8.9 years (4.9 years of trial experience and 4 years of post-trial follow-up $)^{9}$. The finding of higher mortality in trial participants with new-onset heart failure compared to their counterparts without heart failure was noted both in the context of preserved (>4 fold higher risk) and reduced (almost 6 fold higher risk) ejection fraction ${ }^{10}$.

The heart failure diagnoses employed in trials such as SPRINT and ALLHAT have been based on use of rigorous, objective diagnostic criteria that have been validated in prior studies. For example, diagnosis of new-onset heart failure in SPRINT required 1) exclusion of chronic, stable heart failure, 2) fulfillment of multiple specific criteria including acute cardiac decompensation resulting in hospitalization or an emergency department visit with administration of intravenous diuretic or inotropic agents, 3) an appropriate response to treatment, and 4) adjudication by a committee blinded to treatment allocation and specifically trained to recognize the diagnosis. The ALLHAT diagnosis was validated against four other ALLHAT definitions with increasingly stringent requirements, two Framingham Heart Study definitions and a diagnosis made by 11 independent (non-ALLHAT) cardiologists who were blinded to the participant's treatment assignment. There was a high level of concordance between the various definitions, with each yielding similar treatment comparison heart failure results and similar heart failure risk consequences ${ }^{8}$.

In a meta-analysis of 123 randomized controlled trials in which blood pressure was lowered with antihypertensive drug therapy, meta-regression identified a relative risk reduction for heart failure that was proportional to the magnitude of the achieved reduction in systolic blood pressure $(p<0.0001)^{11}$. For a $10 \mathrm{~mm} \mathrm{Hg}$ lower level of systolic blood pressure, the hazard ratio for heart failure was 0.72 ( $95 \% \mathrm{Cl} 0.67$ to 0.78 ). This was independent of starting level of blood pressure and the presence or absence of cardiovascular disease at baseline. Diuretics were superior to all other drug classes for prevention of heart failure, with a hazard ratio of 0.81 ( $95 \% \mathrm{Cl} 0.75$ to 0.88 ), independent of whether the trials included participants with or without heart failure at baseline.

\section{Summary and conclusions}

Heart failure is an increasingly common cardiovascular complication, especially in older adults. It is associated with substantial mortality/morbidity and cost. Blood pressure is the most important preventable risk factor for heart failure and is an underlying cause of other heart failure risk factors such as left ventricular hypertrophy and ischemic heart disease. Antihypertensive drug treatment trials have repeatedly demonstrated that lowering blood pressure prevents heart failure and meta-regression analysis identifies a strong dose-response relationship. Because of its high burden of illness, increasing prevalence, relationship to high blood pressure in observational studies and antihypertensive drug treatment trials, heart failure should be an outcome in event-based randomized controlled trials of blood pressure reduction., especially those conducted in older adults.

\section{Key References}

1. Benjamin EJ, Muntner P, Alonso A, et al. Heart Disease and Stroke Statistics-2019 Update: A Report From the American Heart Association. Circulation. 2019;139(10):e56-e528.

2. Lewington S, Clarke R, Qizilbash N, Peto R, Collins R, Prospective Studies C. Age-specific relevance of usual blood pressure to vascular mortality: a meta-analysis of individual data for one million adults in 61 prospective studies. Lancet. 2002;360(9349):1903-1913.

3. Rapsomaniki E, Timmis A, George J, et al. Blood pressure and incidence of twelve cardiovascular diseases: lifetime risks, healthy life-years lost, and age-specific associations in 1.25 million people. Lancet. 2014;383(9932):1899-1911.

4. Khatibzadeh S, Farzadfar F, Oliver J, Ezzati M, Moran A. Worldwide risk factors for heart failure: a systematic review and pooled analysis. Int J Cardiol. 2013;168(2):1186-1194.

5. Veterans Administration Cooperative Study Group. Effects of treatment on morbidity in hypertension. II. Results in patients with diastolic blood pressure averaging 90 through 114 mm Hg. JAMA. 1970;213(7):1143-1152.

6. Beckett NS, Peters R, Fletcher AE, et al. Treatment of hypertension in patients 80 years of age or older. N Engl J Med. 2008;358(18):1887-1898. 7. Upadhya B, Rocco M, Lewis CE, et al. Effect of Intensive Blood Pressure Treatment on Heart Failure Events in the Systolic Blood Pressure Reduction Intervention Trial. Circ Heart Fail. 2017;10(4). 
8. Einhorn PT, Davis BR, Massie BM, et al. The Antihypertensive and Lipid Lowering Treatment to Prevent Heart Attack Trial (ALLHAT) Heart Failure Validation Study: diagnosis and prognosis. Am Heart J. 2007;153(1):42-53.

9. Piller LB, Baraniuk S, Simpson LM, et al. Long-term follow-up of participants with heart failure in the antihypertensive and lipid-lowering treatment to prevent heart attack trial (ALLHAT). Circulation. 2011;124(17):1811-1818.

10. Davis BR, Kostis JB, Simpson LM, et al. Heart failure with preserved and reduced left ventricular ejection fraction in the antihypertensive and lipid-lowering treatment to prevent heart attack trial. Circulation. 2008;118(22):2259-2267.

11. Ettehad D, Emdin CA, Kiran A, et al. Blood pressure lowering for prevention of cardiovascular disease and death: a systematic review and meta-analysis. Lancet. 2016;387(10022):957-967.

Figure legend: Risk of all-cause mortality and selected cardiovascular disease complication during trial follow-up in Systolic Blood Pressure Intervention Trial (SPRINT) participants with and without incident heart failure (HF). CV = cardiovascular; MI ACS = myocardial acute coronary syndrome. Adapted, with permission, from Upadhya B et al. Clin Heart Fail. 2017;10:e003613.

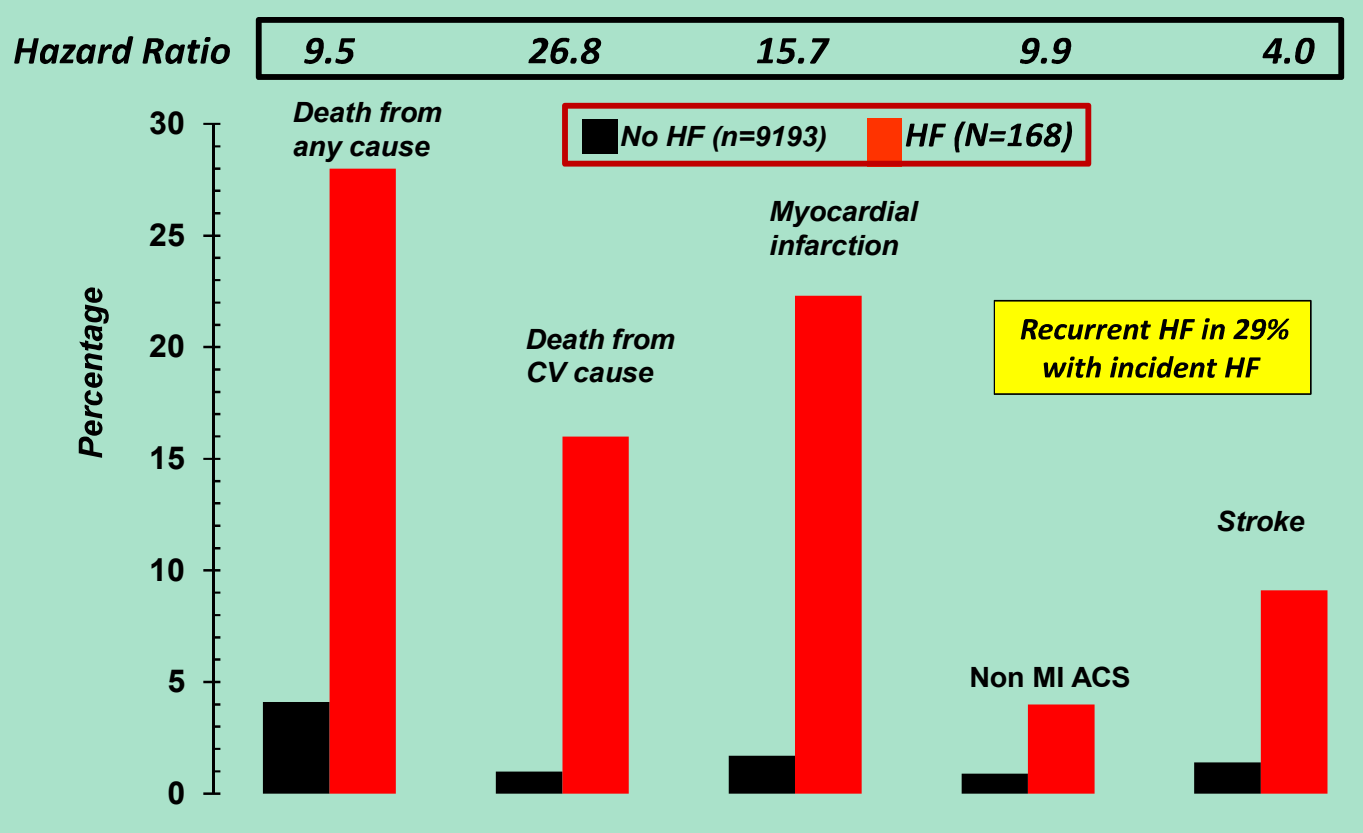

Table. Heart failure experience in six trials of antihypertensive drug treatment

\begin{tabular}{|l|l|l|l|l|l|l|l|}
\hline Trial* & Year & Size & Design** & Active & Control & $\begin{array}{l}\text { Age, years } \\
\text { (mean) }\end{array}$ & $\begin{array}{l}\text { Heart failure } \\
\text { (active vs. control) }\end{array}$ \\
\hline $\begin{array}{l}\text { (active vs. } \\
\text { control) }\end{array}$ & & & & & & & \\
\hline EWPHE & 1985 & 840 & DB & HCTZ*** & Placebo & $\geq 60(--)$ & $-63 \%$ \\
\hline SHEP & 1991 & 4736 & DB & Chlorthalidone & Placebo & $\geq 60(72)$ & $-49 \%$ \\
\hline Syst-Eur & 1997 & 4695 & DB & Nitrendipine & Placebo & $\geq 60(70)$ & $-29 \%$ \\
\hline Syst-China & 1998 & 2394 & Alternative & Nitrendipine & Placebo & $\geq 60(66)$ & $-58 \%$ \\
\hline HYVET & 2008 & 3845 & DB & Indapamide & Placebo & $\geq 80(83)$ & $-64 \%$ \\
\hline SPRINT & 2015 & 9361 & Open & Intensive & Standard & $\geq 50(68)$ & $-38 \%$ \\
\hline & & & & & & & \\
\hline
\end{tabular}

*EWPHE=European Working Party on high Blood Pressure in the Elderly Trial; SHEP=Systolic Hypertension in the Elderly Program; Syst-Eur=Systolic Hypertension in Europe Trial; Syst-China=Systolic Hypertension in China Trial; HYVET=Hypertension in the Very Elderly Trial; SPRINT=Systolic Blood Pressure Intervention Trial.

**DB=double-blind; Alternative=alternative allocation to active treatment or placebo; Open=random allocation to more intensive (Intensive) or less intensive (Standard) treatment

***Hydrochlorothiazide combined with triamterene 